\title{
Microfiber Sagnac Interferometer for Sensing Applications
}

\author{
Michal Kuczkowski, ${ }^{* 1,2}$ Cui Ying, ${ }^{2,3}$ Xuan Quyen Dinh, ${ }^{2,4}$ Perry Ping Shum, ${ }^{3}$ Katarzyna A. Rutkowska, \\ and Tomasz R. Wolinski ${ }^{1}$ \\ ${ }^{1}$ Faculty of Physics, Warsaw University of Technology, ul. Koszykowa 75, 00-662 Warszawa, Poland \\ ${ }^{2}$ CINTRA CNRS/NTU/THALES, UMI 3288, Research Techno Plaza, 50 Nanyang Drive, 637553 Singapore, \\ ${ }^{3}$ School of EEE, Nanyang Technological University, 50 Nanyang Avenue, 639798 Singapore \\ ${ }^{4}$ Thales Solutions Asia Pte Ltd, R\&D Department, 28 Changi North Rise, 498755 Singapore
}

Received November 22, 2012; accepted December 20, 2012; published December 31, 2012

\begin{abstract}
In this paper we propose an optical fiber-based refractive index and temperature sensor using an MNF (micro/nano fiber) inserted in a Sagnac loop interferometer [1]. A portion of the PMF is tapered into a micro/nano dimension to increase the interactions between a guided signal and the surrounding environment. Experimental tests have demonstrated low response to temperature and high sensitivity to refractive index change of a liquid surrounding the MNF. It has been shown that the sensitivity of the setup under test to temperature decreases when a longer MNF is used. The maximum sensitivity for a refractive index achieved equals to $1068 \mathrm{~nm} / \mathrm{RIU}$, which was obtained by a $4.7-\mathrm{mm}$ long MNF with a diameter of $30 \mu \mathrm{m}$.
\end{abstract}

The Sagnac interferometer is a double-beam interferometer in which the light from a source is split by a $50 \%$ transparent plate into two beams of equal power. One beam is the reference and the other (called the signal beam) carries the information. The beams follow the same trajectory but in the opposite directions. When the beams reach a coupler they interfere with each other. By changing the phase of the signal beam, or by changing its optical path (e.g. due to a change in the refractive index or geometrical path), it is possible to alter the interference pattern $[1,2]$.

The experimental setup shown in Fig. 1 consists of a 3$\mathrm{dB}$ coupler connected to a polarization-maintaining fiber (PMF). The MNF is located in a section of the PMF and is used as a sensing part in the Sagnac interferometer.

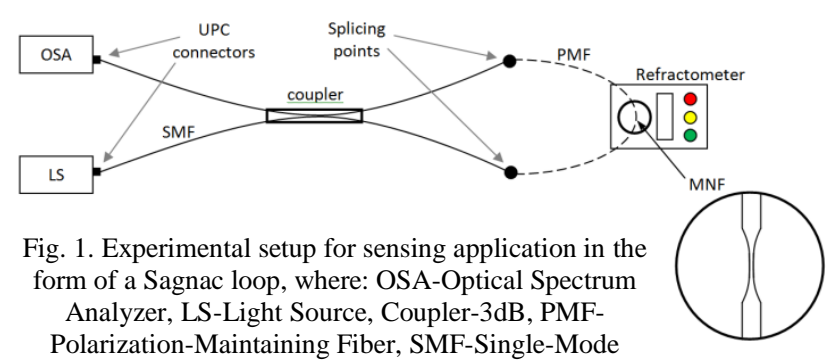
Fiber.

The 3-dB coupler acts as a 50:50 beam splitter. A relatively high birefringent $\mathrm{PMF}$ employed here introduces optical path differences between two counterpropagating beams and causes fringes in the

\footnotetext{
*E-mail: kuczkowski@if.pw.edu.pl
}

output spectrum. By tapering the PMF, part of the light power leaks out and interacts with the vicinity, making the Sagnac loop particularly sensitive to some environmental changes. The birefringence of the PMF is related to its asymmetrical core of the PMF; it is contrary to the traditional SMF with a circular core for which the same values of effective refractive indices in all transversal directions are observed.

The transmittance of the Sagnac loop can be approximated by a periodic function of the wavelength in terms of phase difference and described as:

$$
T=\frac{1-\cos (\psi)}{2}
$$

where $\psi$ is the phase given by:

$$
\psi=\frac{2 \pi}{\lambda} L_{0} B
$$

with $\lambda$ - the operating wavelength, $L_{0}$ - the length of the fiber and $B$ - the birefringence. The wavelength spacing $S$ between the adjacent transmission notches or peaks is given by:

$$
S=\frac{\lambda^{2}}{B L_{0}} .
$$

Any refractive index change in the medium surrounding the fiber causes variation in birefringence $\Delta B$, which is the main contribution to the phase difference. Therefore the equation for the phase difference can be described as:

$$
\Delta \psi=\frac{2 \pi}{\lambda} L \cdot \Delta B
$$

where $L$ is the sensing length (i.e. MNF length).

The phase difference induces the change in the spectral position of the notch (or peak) in the Sagnac output spectrum and this spectral shift is given by:

$$
\Delta \lambda=\frac{S \Delta \psi}{2 \pi}=\frac{\left(\frac{\lambda^{2}}{B L_{0}} \frac{2 \pi}{\lambda} L \cdot \Delta B\right)}{2 \pi},
$$

where

$$
\Delta \lambda=\lambda \frac{\Delta B}{B} \frac{L}{L_{0}}
$$

and $L / L_{0}$ is the ratio between sensing part and total length of PMF [3, 4]. 
It means that the information about the change in the birefringence $\left(\Delta B=\Delta n_{\mathrm{s}}-\Delta n_{\mathrm{f}}\right.$, where $n_{\mathrm{s}}$ and $n_{\mathrm{f}}$ are refractive indices of slow and fast axis of PMF, respectively) can be obtained by measuring the shift of the notch in the output spectrum. In particular, it can be used to control the change in the refractive index around the fiber forming the Sagnac loop. While changes in the effective refractive index are different for slow and fast axes, a variation in the birefringence is obtained. This, in turn, results in a shift in the output spectrum, as mentioned before.

It is important to note that in the setup proposed, it is necessary to taper the fiber in the Sagnac loop (i.e. to make the fiber diameter smaller) in order to make it more sensitive for refractive index changes. Moreover, when the fiber with a small diameter is used, any liquid placed around the fiber stays closer to the core area, which ensures higher influence on the effective indices (for both fast and slow axes).

In order to study the location of notches in the spectral range under consideration, a solution of glycerin (with a refractive index of 1.47) and demineralized water was used. An increased concentration of glycerol allows liquids with different refractive indices to be obtained. Temperature dependence was analyzed in pure water.

Using the experimental setup from Fig. 1, in addition to several probes containing substances with different refractive indices, a typical shift of the notch in the transmission spectrum towards longer wavelengths is obtained when the refractive index is increased, as shown in Fig. 2. It is important to note that the output power level for the transmission spectrum remains unchanged for different refractive indices investigated.

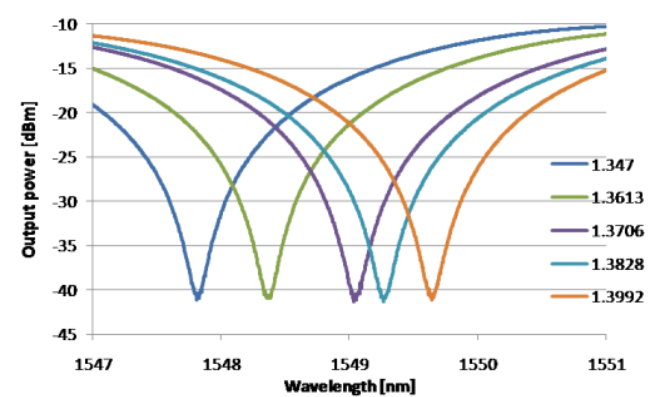

Fig. 2. Application of the Sagnac loop composed of the PMF (with $\mathrm{MNF}$ ) for refractive index sensing - position of the notch in the transmission spectrum depends on the refractive index of the substance under tests.

In the first case shown in Fig. 3a, a sensitivity of $35.84 \mathrm{~nm} / \mathrm{RIU}$ (refractive index unit) can be gathered from the linear fit performed. The coefficient of determination $\left(\mathrm{R}^{2}\right) 0.966$ is pretty high showing a good linear fitting. In the second case, when the experiment is repeated using a longer sample (see Fig. 3b), a slightly higher coefficient of determination is obtained $\left(\mathrm{R}^{2}=0.973\right)$, accompanied by a higher sensitivity of $1068 \mathrm{~nm} / \mathrm{RIU}$. The results obtained are significantly high when compared to the results of other methods: for example the photonic crystal fiber tip (11.5nm/RIU) [6], the higher-order mode reflection of a microfiber Bragg grating (102nm/RIU) [7], MachZehnder interferometer formed by three cascaded singlemode fiber tapers $(28.6 \mathrm{~nm} / \mathrm{RIU})$ [8], or supported microfiber loops $(17.8 \mathrm{~nm} / \mathrm{RIU})$ [9].

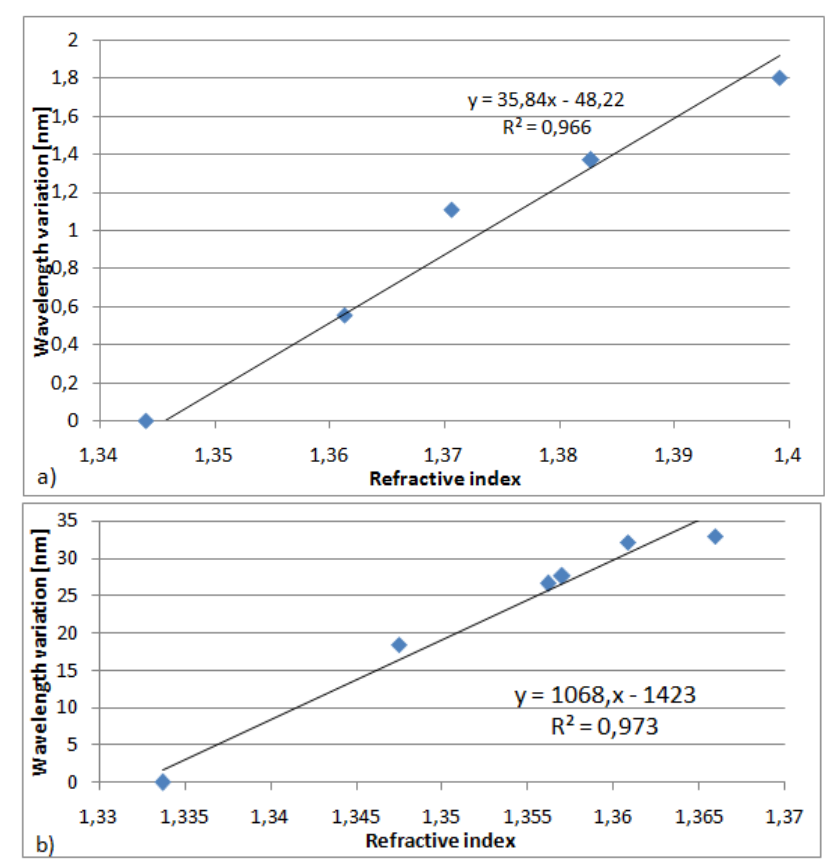

Fig. 3. Application of the Sagnac loop for refractive index sensing dependence of the position of the notch in the transmission spectrum on the refractive index of the substance placed around the fiber. The parameters of fibers under tests are: a) $60 \mu \mathrm{m}$ in diameter and $130 \mu \mathrm{m}$ in length b) $30 \mu \mathrm{m}$ in diameter and $4.7 \mathrm{~mm}$ in length.

In both cases presented above, the coefficient of correlation $\left(\mathrm{R}^{2}\right)$ close to one confirms linear dependence of the spectral position of notches in transmission. Higher sensitivity for refractive index changes obtained in this case (shown in Fig. 3b) is related to: (i) longer length and (ii) smaller cross section of the PM MNF which implicate stronger influence exerted by environmental conditions. It causes the effect of optical birefringence changes to be stronger and therefore easier to be observed.

As mentioned before, when longer section of MNF is tapered [2], the system sensitivity to a refractive index change in the surrounding environment of the sentient element significantly increases. In our case it means that the optimal condition for the detection is to fabricate a long section of the MNF, then place it into a liquid. The impact of the liquid for a long section of the MNF (i.e. longer than this previously fabricated with use of $\mathrm{CO}_{2}$ laser [5]) allows very small changes in the refractive index of the liquid to be detected. With the sensitivity over $1000 \mathrm{~nm} / \mathrm{RIU}$ and the spectral resolution of $0.02 \mathrm{~nm}$, it should be possible to sense the refractive index changes as low as $2.7 * 10^{-5}$ RIU. However, it seems that such a high sensitivity of the test system is very difficult if not 
impossible to be obtained in practice. That is related to the liquid density fluctuations in different areas of its volume and its constant diffusion. Those changes induce noise in the measurements of the notches position and also decrease the measurement resolution.

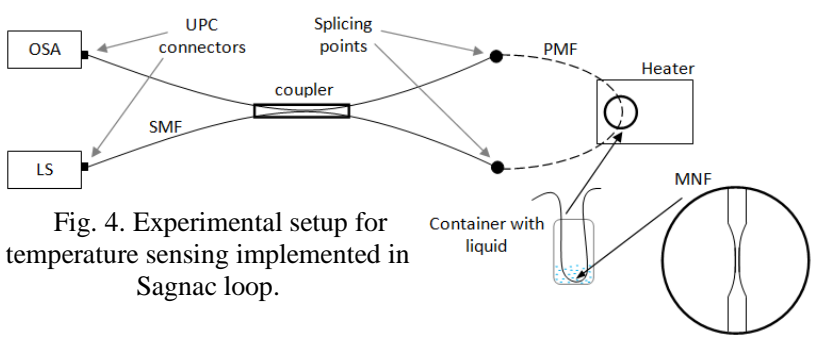

While the PANDA PMF is particularly sensitive for the temperature changes [10], this type of fiber was applied in the setup shown in Fig. 4. In current experiment demineralised water was used. Obviously, refractive index of the liquid under tests (demineralised water) varies with temperature, but a refractive index change of about $0.00016 \mathrm{RIU} /{ }^{\circ} \mathrm{C}$ can be neglected. The shift in the notch position induced by temperature change equal to $0.0215 \mathrm{~nm} /{ }^{\circ} \mathrm{C}$ is relatively small compared to refractive index change. Temperature sensitivity (as shown in Fig. 5a) goes down with decreasing fiber diameters (Fig. 5b).

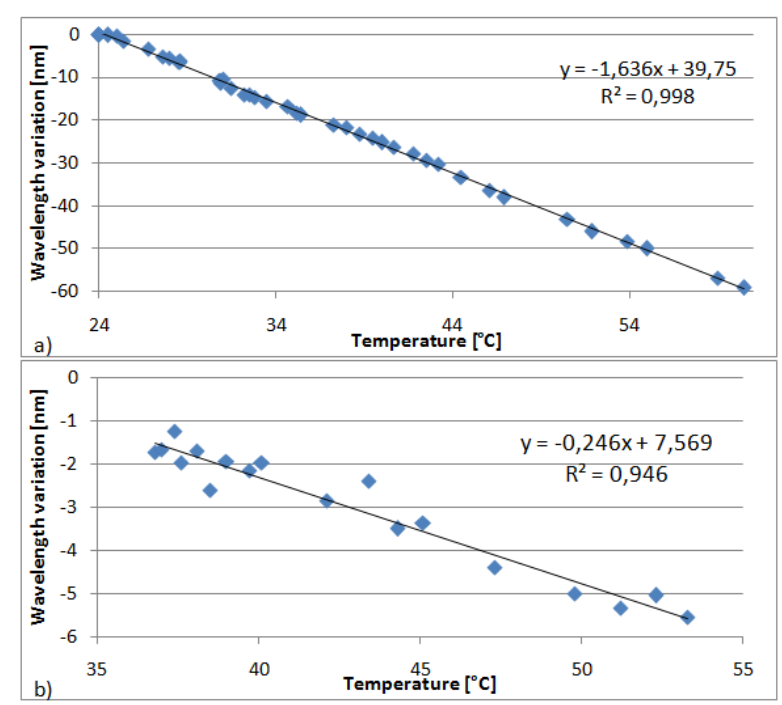

Fig. 5. Temperature dependence of the notch position for a MNF with a) $70 \mu \mathrm{m}$ in diameter and $100 \mu \mathrm{m}$ in length, b) $30 \mu \mathrm{m}$ in diameter and $4.5 \mathrm{~mm}$ in length.

As shown in Fig. 5a, sensitivity of $1.6 \mathrm{~nm} /{ }^{\circ} \mathrm{C}$ obtained here is quite similar to the result obtained with use of a traditional PANDA fiber (i.e. without part of the MNF as applied here) $[10,11]$. At the same time temperature sensitivity for this fiber varies from $0.9 \mathrm{~nm} /{ }^{\circ} \mathrm{C}$ to $1.9 \mathrm{~nm} /{ }^{\circ} \mathrm{C}$. When the MNF is tapered on a length of about $15 \mathrm{~mm}$ (that is much longer than in the fibers previously studied), with $30-40 \mu \mathrm{m}$ in diameter, significant drop in the temperature sensitivity is observed. As shown in Fig. $5 \mathrm{~b}$, the notch position varies with increasing temperature.

Sensitivities of $1.636 \mathrm{~nm} /{ }^{\circ} \mathrm{C}$ (Fig. 5a) and $0.246 \mathrm{~nm} /{ }^{\circ} \mathrm{C}$ (Fig. 5b) obtained here are six times weaker than the one of a short MNF fabricated by a $\mathrm{CO}_{2}$ laser, (for which $1.5 \mathrm{~nm} /{ }^{\circ} \mathrm{C}$ was obtained). Moreover the results do not show the high linear dependence anymore which is confirmed by the low correlation coefficient $(\sim 0.9)$.

The reason of the inferior linear dependence obtained here (closely related to the multimode operation) is the diameter size of $30 \mu \mathrm{m}$. It introduced noise in the measurement. We could say that for optical fibers of small diameters, temperature sensitivity is low or simply negligible. However, further research needs to be conducted to prove it.

In conclusion, we have demonstrated a fiber Sagnac interferometer applied used as a sensor for two kinds of impacts, namely - temperature and refractive index changes of the liquid surrounding optical MNFs. A shortlength of the PMF with an MNF was utilized as a sensing element. A simplified analytical description for the spectrum shift in response to a temperature change has been derived. The sensor setup with a sensitivity of $1.9 \mathrm{~nm} /{ }^{\circ} \mathrm{C}$ was obtained for a standard $125 \mu \mathrm{m}$ fiber, and a cdecreasing value of $1.6 \mathrm{~nm} /{ }^{\circ} \mathrm{C}$ for $\mathrm{MNF}$ with $70 \mu \mathrm{m}$ in diameter and $100 \mu \mathrm{m}$ in length. For smaller diameters of MNF, sensitivity drops to $0.27 \mathrm{~nm} /{ }^{\circ} \mathrm{C}$ for MNF with $30 \mu \mathrm{m}$ in diameter and $4500 \mu \mathrm{m}$ in length. Our layout shows the opposite dependence for the changes of refractive index. The sensitivity for refractive index changes in the case of a short piece of the MNF (shorter than $500 \mu \mathrm{m}$ ) was less than $200 \mathrm{~nm} / \mathrm{RIU}$, while for a long MNF with a length of $4700 \mu \mathrm{m}$ maximum sensitivity of $1068 \mathrm{~nm} / \mathrm{RIU}$ is obtained. The refractive index sensitivity of our setup is higher than the one obtained in the systems with a PCF, microfiber Bragg grating, Mach-Zehnder interferometer, microfiber loops [6-9]. The RI sensitivity is as high as in the micro/nanofiber Bragg grating but with lower temperature sensitivity. In future work, it is essential to check whether temperature dependence can be completely eliminated with the use of a long nanofiber for which the refractive index sensitivity should increase.

\section{References}

[1] G. Wang, P.P. Shum, L. Tong, C.M. Li; C. Lin, IEEE Phot. Tech. Lett. 22, 586 (2010).

[2] L. M. Tong, M. Sumetsky, Subwavelength and Nanometer Diameter Optical Fibers (Zhejiang University Press, Springer 2009).

[3] H. Gong, C.C. Chan, L. Chen, X. Dong, IEEE Phot. Tech. Lett. 22(16), 1238 (2010).

[4] X. Dong, H. Tam, P. Shum, Appl. Phys. Lett. 90, 151113 (2007).

[5] C. E. Chryssou, Opt. Eng. 38(10), 1645 (1999).

[6] K. Milenko et al., Opt. Lett. 37(8), 1373 (2012).

[7] Y. Zong et al., Opt. Exp. 18(25), 26345 (2010).

[8] D. Wu et al., Appl. Opt. 50(11), 1548 (2011).

[9] X. Guo, L. Tong, Opt. Exp. 16(19), 14429 (2008).

[10] J. Zhan et al., J. Lightwave Technol. 29(24), 3640 (2011).

[11] O. Frazão, J.M.T. Baptista, J.L. Santos, Sensors 7, 2970 (2007). 